\title{
Atomic layer deposition-A novel method for the ultrathin coating of minitablets
}

\section{Hautala, Jaana}

2017-10-05

Hautala , J , Kääriäinen , T, Hoppu , P , Kemell , M , Heinämäki , J , Cameron , D , George , S \& Juppo , A M 2017 , ' Atomic layer deposition-A novel method for the ultrathin coating of minitablets ' , International Journal of Pharmaceutics , vol. 531 , no. 1 , pp. 47-58 . https://doi.org/10.1016/j.ijpharm.2

http://hdl.handle.net/10138/309237

https://doi.org/10.1016/j.jpharm.2017.08.010

cc_by_nc_nd

acceptedVersion

Downloaded from Helda, University of Helsinki institutional repository.

This is an electronic reprint of the original article.

This reprint may differ from the original in pagination and typographic detail.

Please cite the original version. 
1 Atomic Layer Deposition - A Novel Method for the Ultrathin Coating of 2 Minitablets

3 Jaana Hautala ${ }^{\mathrm{a}, *}$, Tommi Kääriäinen ${ }^{\mathrm{b}, \mathrm{c}, \mathrm{d}}$, Pekka Hoppu ${ }^{\mathrm{c}}$, Marianna Kemell ${ }^{\mathrm{d}}$, Jyrki Heinämäki ${ }^{\mathrm{e}}$,

4 David Cameron $^{\mathrm{f}}$, Steven George ${ }^{\mathrm{b}}$, Anne Mari Juppo ${ }^{\mathrm{a}}$

5 a Industrial Pharmacy, Faculty of Pharmacy, University of Helsinki, P.O. Box 56 (Viikinkaari

6 5E, Biocenter 2), FI-00014 University of Helsinki, Finland

7 b Department of Chemistry and Biochemistry, Faculty of Chemistry and Biochemistry,

8 University of Colorado Boulder, 596 UCB, Boulder, CO 80309, USA

$9 \quad{ }^{\mathrm{c}}$ NovaldMedical Ltd Oy, Telkäntie 5, FI-82500 Kitee, Finland

$10{ }^{\mathrm{d}}$ Department of Chemistry, Faculty of Science, University of Helsinki, P.O. Box 55 (A. I.

11 Virtasen aukio 1), FI-00014 University of Helsinki, Finland

$12{ }^{\mathrm{e}}$ Institute of Pharmacy, Faculty of Medicine, University of Tartu, Nooruse 1, EE-50411 Tartu, 13 Estonia

$14{ }^{\mathrm{f} C e p l a n t,}$ Masaryk University, Kotlářská 267/2, CZ-61137 Brno, Czech Republic

$15 *$ Corresponding author at: Formulation and Industrial Pharmacy, Department of Pharmaceutical

16 Chemistry and Technology, Faculty of Pharmacy, University of Helsinki, P.O. Box 56

17 (Viikinkaari 5E, Biocenter 2), FI-00014 University of Helsinki, Finland. Tel.: +358 294159627.

18 E-mail

address:

jaana.hautala@helsinki.fi 


\section{ABSTRACT}

We introduce atomic layer deposition (ALD) as a novel method for the ultrathin coating (nanolayering) of minitablets. The effects of ALD coating on the tablet characteristics and taste masking were investigated and compared with the established coating method. Minitablets containing bitter tasting denatonium benzoate were coated by ALD using three different $\mathrm{TiO}_{2}$ nanolayer thicknesses (number of deposition cycles). The established coating of minitablets was performed in a laboratory-scale fluidized-bed apparatus using four concentration levels of aqueous Eudragit ${ }^{\circledR}$ E coating polymer. The coated minitablets were studied with respect to the surface morphology, taste masking capacity, in vitro disintegration and dissolution, mechanical properties, and uniformity of content. The ALD thin coating resulted in minimal increase in the dimensions and weight of minitablets in comparison to original tablet cores. Surprisingly, ALD coating with $\mathrm{TiO}_{2}$ nanolayers decreased the mechanical strength, and accelerated the in vitro disintegration of minitablets. Unlike previous studies, the studied levels of $\mathrm{TiO}_{2}$ nanolayers on tablets were also inadequate for effective taste masking. In summary, ALD permits a simple and rapid method for the ultrathin coating (nanolayering) of minitablets, and provides nanoscalerange $\mathrm{TiO}_{2}$ coatings on porous minitablets. More research, however, is needed to clarify its potential in tablet taste masking applications.

\section{KEYWORDS}

Atomic layer deposition, thin film coating, $\mathrm{TiO}_{2}$, minitablet, taste masking, polymer film coating

8 ABBREVIATIONS

ALD Atomic layer deposition 
40 MCC Microcrystalline cellulose

41 HPC Hydroxypropyl cellulose

42 SPB Sodium phosphate buffer

43 ACN Acetonitrile

44 HPLC High performance liquid chromatography

45 RT Room temperature

46 RH Relative humidity

47 RSD Relative standard deviation

48 SEM Scanning electron microscope

49 SEM-EDS Scanning electron microscope with energy dispersive spectroscopy

50 1. INTRODUCTION

51 For tablets, polymer film coating is still the most widely used and efficient taste masking

52 technique in the pharmaceutical industry (Ayenew et al., 2009; Joshi and Petereit, 2013; Sohi et

53 al., 2004). In addition to taste masking purposes, polymer film coating of tablets offers a variety

54 of practical advantages that contribute to their therapeutic effect, as well as ensure patient

55 compliance and tablet product stability throughout their shelf life (Bruce et al., 2011; Felton,

56 2007; Joshi and Petereit, 2013; Pearnchob et al., 2003; Siepmann et al., 2013). With polymer

57 film coating, however, sufficient taste masking and drug release control demand relatively thick

58 polymer films, and consequently, the application of large amounts of polymers (Joshi and 
59 Petereit, 2013). The conventional film coating methods are associated also with laborious,

60 expensive and technical expertise demanding material selection and process development, a lot is

61 required also from coated tablet core and coating formulation design (Joshi and Petereit, 2013).

62 Thus, the pharmaceutical industry is continually searching for new effective and inexpensive

63 coating approaches for tablet taste masking and palatability improvement.

64 Atomic layer deposition (ALD) is a surface controlled, self-limiting layer-by-layer proceeding

65 coating method for depositing ultra-thin, high quality and conformal thin films, these even on

66 high aspect ratio structures (George, 2010). ALD is commonly used in microelectronics and

67 nanotechnology applications, in functions, where miniaturization of structures requires the

68 control of film thickness at atomic level (George, 2010; Puurunen, 2005). Consequently, the

69 important features of ALD regard surface protection, modification, and functionalization, these

70 in a number of applications benefiting from the characteristics of ALD (George, 2010). In

71 pharmaceuticals, the moisture protective element of ALD coating has been already utilized for

72 nano- and micro-sized drug particles (Carlsson et al., 2016). The ALD technique has been

73 successfully applied also in the design of larger-sized, single particles intended for

74 pharmaceutical powder applications (Hoppu et al., 2015). Here, the present situation is more than

75 inspiring, since the thin film technology combined with material science could lead to new

76 pharmaceutical manufacture and formulation options for fabricating the protective coatings on

77 single drug particles. Bringing such approach to life science markets to serve the evolving needs

78 of drug development, formulation engineering and manufacturing processes could hold great

79 promises on homogeneous drug particles, the creation of new and tailor-made solid dosage

80 forms, improvements in handling moisture sensitive and electrically charged single drug

81 particles, and a decrease in the number of excipients and manufacturing process steps. In 
82 addition to pure drug substances and single particles, ALD coatings could be also applicable for

83 larger, more complex shaped, porous and heterogeneous substrates, such as tablets. The approach

84 seems attractive, as ALD coatings are not only continuous, ultra-thin, dense, and smooth, but

85 also most importantly pinhole-free, very conformal to the substrate, and provide good diffusion

86 barriers with low gas and moisture permeability (George, 2010). In addition, the thin ALD

87 coatings can reach and fill the surface of even the deepest and narrowest voids and pores (Ritala

88 and Leskelä, 2002). Therefore, from the pharmaceutical product design and industry point of

89 view, ALD would provide a very exciting technology not only for drug particle design but also

90 as a potential method for tablet coating purposes.

91 ALD nanoscale films are formed (grown through deposition) utilizing self-limiting chemical

92 reactions between gaseous precursors chemisorbed on a solid substrate surface. For example,

93 when depositing metal oxide films, compounds of zinc, aluminum or titanium can be used as the

94 metal precursors and water as the oxygen precursor. In many cases, the ALD growth starts by

95 chemisorption of the metal precursor molecules on the hydroxyl groups of the surface. Tablets

96 should allow the initiation of the nanolayering process of ALD, as they are commonly composed

97 of multiple organic excipients containing free hydroxyl groups. In tablets, the number of surface

98 hydroxyl groups required for attachment of molecules can even be created with the small amount

99 of surface moisture readily existing on tablet surfaces and pores. Moreover, from a possible

100 tablet coating point of view, the chemical components like zinc, aluminum and titanium oxides

101 applied by ALD are all established pharmaceutical excipients, and thus their compatibility and

102 safety profiles are well known. Also, compared to the conventional polymer film coating, ALD

103 process is very different. The established polymer coating, performed by a fluidized-bed

104 technique, sets high demands for tablet core strength, and consequently, for tablet formulation 
105 design. During polymer coating in a fluidized-bed, tablets are exposed to frequent collisions, 106 high friction, increased moisture and high temperature. Consequently, tablet cores with limited 107 hardness and mechanical strength are eroded and broken down, especially in the beginning of the 108 coating process until the uniform film coating is formed through concomitant moisture 109 evaporation and polymer particle coalescence (Lehmann, 1997; Mehta, 1997). Thin coating by 110 ALD does not involve such limitations. In flow type ALD reactor, tablet cores are stationary, and 111 are coated during separate surface saturation (deposition) cycles involving chemical interactions 112 (Fig. 1). In theory, in ALD involving metal oxides, a cycle of surface saturation takes place 113 through two reaction steps performed commonly in vacuum at controlled temperature. During 114 step one (the first half cycle) the metal precursor such as $\mathrm{TiCl}_{4}$ vapor, pulsed to the coating 115 chamber, is allowed to react with the free hydroxyl groups on the substrate surface. Therefore, in 116 theory, the chemical pulse saturates the surface with Ti containing groups through molecular 117 bonds. During step two (the second half cycle) the molecularly bonded structures on the 118 substrate surface react with the oxygen precursor (water vapor) and form the first nanolayer of $119 \mathrm{TiO}_{2}$. Between these steps, a purge of inert gas $\left(\mathrm{N}_{2}\right)$ is applied in order to remove any excess of 120 precursor and the reaction by-products. The thickness of the films can be controlled by repeating 121 the number of reaction cycles to reach the desired coating thickness. Moreover, compared to the 122 established polymer coating, the ALD process can be completely pre-programmed, and it does 123 not involve high labor or coating raw material related costs.

124 To date, no well-documented or established study to evaluate the feasibility of ALD for thin 125 coating of tablets has been performed. Lehtonen and co-workers (2013) have evaluated ALD on 126 simple and single material layered tablets, but as pharmaceutical solids traditionally are 127 heterogeneous and complex substrates, a need for a more thorough study on the applicability of 
ALD on tablets is evident. Moreover, taste and odor masking capacity of ALD has been studied only on fish oil containing soft gel capsules (Lehtonen et al., 2013). Therefore, we are also completely lacking of information on the applicability and capacity of such ALD ultrathin coatings to improve palatability and taste masking associated with tablets.

Here, we therefore introduce our study describing ALD on minitablets composed of multiple excipients and a bitter tasting model drug. We investigated ALD as a novel technique for thin coating of minitablets, and for masking the bitter taste of model substance of denatonium benzoate. Special attention was paid to the effects of the ALD nanolayering process on the minitablet properties and taste masking efficacy. An established bottom-spray fluidized-bed coating (with a Wurster column set-up) was used as a reference coating technique.

\section{MATERIALS AND METHODS}

\subsection{Materials}

Granules were prepared using denatonium benzoate (Sigma-Aldrich, China) as a model substance of a bitter tasting active drug ingredient $(0.04 \% \mathrm{~m} / \mathrm{m})$. Microcrystalline cellulose (MCC) (Avicel $^{\circledR}$ PH-102, FMC Corporation, Ireland) $(63 \% \mathrm{~m} / \mathrm{m})$ and calcium hydrogen phosphate dihydrate (Emcompress ${ }^{\circledR}$, Albright \& Wilson, Australia) $(30 \% \mathrm{~m} / \mathrm{m})$ were used as fillers, and hydroxypropyl cellulose (HPC) (Klucel ${ }^{\circledR}$ JXF, Aqualon France SA, France) (3\% $\mathrm{m} / \mathrm{m}$ ) and crospovidone (Kollidon ${ }^{\circledR}$ CL-F, BASF Corporation, Germany) $(4 \% \mathrm{~m} / \mathrm{m}$ ) as a binder and disintegrant, respectively. Sodium stearyl fumarate (Pruv ${ }^{\circledR}$, JRS Pharma, Spain) $(1 \%$ m/m) was added as a lubricant before tableting.

The synthetic copolymer based on butyl methacrylate, (2-dimethylaminoethyl) methacrylate and methyl methacrylate (1:2:1) Eudragit $^{\circledR}$ E PO, Evonik Industries AG, Germany) was used as a 
150 film forming and taste masking polymer in film coating of minitablets. Lutrol ${ }^{\circledR} \mathrm{F} 127$ (poloxamer 151 407, BASF, Germany) (10\% m/m, on dry polymer) and dibutyl sebacate (Fluka Chemie AG, 152 Germany) $(15 \% \mathrm{~m} / \mathrm{m}$, on dry polymer) were used as the dispersing agent and plasticizer, 153 respectively. Magnesium stearate, Ph.Eur. (donated by Orion Pharma, Finland) was used as an 154 antitacking agent $(35 \% \mathrm{~m} / \mathrm{m}$, on dry polymer). Distilled water was used as a dispersion medium 155 for film coating.

$156 \mathrm{ALD}_{\mathrm{TiO}}$ films were grown from titanium tetrachloride $\left(\mathrm{TiCl}_{4}\right)$ (Sigma-Aldrich, USA) and 157 deionized water. $\mathrm{TiO}_{2}$ was chosen over zinc and aluminium oxides due to its common use as a 158 coloring agent in pharmaceutical coating formulations. The $\mathrm{TiO}_{2}$ was considered beneficial also 159 for taste masking purpose due to its hydrophobic (water insoluble) nature.

160 Distilled water was used as a test medium for tablet disintegration in vitro. The in vitro 161 dissolution tests were performed using sodium phosphate buffer (SPB) pH 7.6 (US 162 Pharmacopoeia) as a testing medium. The SPB was composed of sodium hydroxide (VWR 163 International S.A.S., France), $\mathrm{KH}_{2} \mathrm{PO}_{4}$ (Riedel-de Haën, Germany) and distilled water. The 164 uniformity of content test of minitablets was also performed with SPB (pH 7.6).

165 Acetonitrile (ACN) (Sigma-Aldrich Chemie GmbH, Germany) and aqueous 10mM ammonium 166 acetate $\mathrm{pH} 4.5$ (Sigma-Aldrich Chemie GmbH, Germany) were used as eluents for high 167 performance liquid chromatography (HPLC) analyses conducted for the dissolution and 168 uniformity of content tests.

\subsection{Design of experiments}

170 Three different number of coating cycles (100, 300 and 500 cycles) resulting different coating 171 thicknesses were used for the ALD thin coating of minitablets. The numbers of cycles were 
172 selected based on the results of preliminary experiments on ALD. For the polymer coating of

173 minitablets, the design of experiments involved four different polymer coating concentrations of

$1742,4,6$ and $8 \mathrm{mg} / \mathrm{cm}^{2}$. The levels were selected based on the existing knowledge on the minimum

175 coating thickness of Eudragit ${ }^{\circledR} \mathrm{E}\left(1-2 \mathrm{mg} / \mathrm{cm}^{2}\right)$ effective enough for taste masking (Evonik, 176 2009).

\section{$177 \quad 2.3$ Preparation of granules and minitablets}

178 Wet granulations of powder mixtures (400 g) were performed in a Diosna high-shear granulator

179 (Dierks\&Söhne GmbH, Germany) using distilled water as a granulating liquid. The mixing 180 speeds for impeller and chopper were $600 \mathrm{rpm}$ and $1500 \mathrm{rpm}$, respectively. The water spraying 181 rate of $140 \mathrm{~g} / \mathrm{min}$ was used. MCC, calcium hydrogen phosphate dihydrate, HPC and 182 crospovidone were first dry mixed for two minutes. Then, the aqueous solution of denatoniun 183 benzoate was pipetted onto the surface of mixed dry powder bed, and subsequently distilled 184 water was sprayed while continuously mixing a powder blend. When the total amount of water $185(0.45 \mathrm{~g} / \mathrm{g})$ was added, granulation was stopped without any kneading phase. The total amount of 186 water was empirically determined based on the preliminary granulation experiments.

187 Wet granules were wet-sieved (Quadro Comil, Quadro Engineering, Canada) and tray-dried at $18840^{\circ} \mathrm{C}$ for 2 hours, and subsequently at room temperature (RT) for overnight. Prior to tablet 189 compression, the dried masses were sieved (Quadro Comil) with a mesh size of $800 \mu \mathrm{m}$. 190 Lubricant (sodium stearyl fumarate) was added and mixed with the granules (Turbula, Willy A. 191 Bachofen AG, Switzerland) for 5 minutes. The masses intended for minitablet compression were 192 allowed to equilibrate at RT/60\% RH (relative humidity) for at least 12 hours before tableting. 
193 The granulated masses were tableted at RT/50\% RH with a rotary tablet press (Ronchi, Officine 194 Meccaniche F.lli Ronchi, Italy) and single tip punches of $3 \mathrm{~mm}$ in diameter to receive round and 195 biconvex minitablets with a target weight of $25 \mathrm{mg}$ and denatonium benzoate strength of $10 \mu \mathrm{g}$. 196 Tablets were produced in three separate batches. The average upper and lower punch 197 compression forces (and relative standard deviations, RSD) for the batches I-III were as follows:

198 Batch I $1.8 \mathrm{kN}(10.3 \%) / 0.7 \mathrm{kN}$ (27.1\%); Batch II $2.0 \mathrm{kN}(11.9 \%) / 1.8 \mathrm{kN}(13.8 \%)$; and Batch 199 III $1.7 \mathrm{kN}(10.6 \%) / 1.5 \mathrm{kN}(11.6 \%)$.

\subsection{Polymer film coating of minitablets}

201 The aqueous polymer coating dispersions were prepared by adding emulsifier and plasticizer into 202 a small portion of distilled water and mixing with a magnetic stirrer. Next, the film-forming 203 polymer was progressively added into the aforementioned solution and mixed with a magnetic 204 stirrer for 30 minutes. Magnesium stearate was then homogenously suspended in the remaining 205 portion of water for 30 minutes with a high-shear mixer (Ultra-Turrax, IKA, Germany). The magnesium stearate suspension was added to the polymer dispersion, and the mixture was

207 rapidly homogenized with a high-shear mixer. Finally, the coating suspension was passed 208 through a 500- $\mu \mathrm{m}$ sieve. The final coating dispersions were continuously mixed for overnight 209 with a magnetic stirrer prior to film coating process.

210 Polymer coating of minitablets was performed in a laboratory-scale fluidized-bed apparatus 211 (Aeromatic AG, Switzerland) equipped with bottom spray-installed Wurster set-up. The height 212 of the Wurster column was $7.0 \mathrm{~cm}$. The nozzle was a Schlick 970/7-1 pneumatic external mixing 213 two-fluid nozzle (Düsen-Schlick GmbH, Germany). The coating processor with instrumentation 214 was connected to a PC and operated via InTouch -software (Wonderware, USA). The coating 
215 batch size was $100 \mathrm{~g}$. The atomizing air pressure was $1.1 \mathrm{bar}$ and the inlet air volume (air flow

216 rate) $12.5 \mathrm{l} / \mathrm{s}$. All coatings were performed in an ambient inlet air $\mathrm{RH}$ of $22 \pm 0.4 \%$ measured

217 with a Vaisala HUMICAP ${ }^{\circledR}$ HMT100 humidity and temperature probe (Vaisala Oyj, Finland).

218 Before each coating experiment, the coating chamber (made of glass) was preheated with an inlet

219 air flow rate of $12.5 \mathrm{l} / \mathrm{s}$, inlet air temperature of $40-50^{\circ} \mathrm{C}$, and outlet air temperature of 220 approximately $40^{\circ} \mathrm{C}$. The main parameters for nozzle diameter, atomizing air pressure, inlet air 221 volume, inlet air temperature and spraying rate describing the actual coating process are given in

222 Table 1. The end-point of a spraying phase was determined as the point where the theoretical 223 polymer amount of 2, 4, 6 and $8 \mathrm{mg} / \mathrm{cm}^{2}$ was achieved. The end-point of a drying phase was 224 reached when the difference in $\mathrm{RH}$ between the inlet and outlet air was constant. Coated 225 minitablets were further tray-dried and cured at $40^{\circ} \mathrm{C}$ for 24 hours.

\subsection{ALD coating of minitablets}

227 The ALD was performed in a laboratory-scale flow type ALD reactor (Beneq TFS 200, Finland).

228 Uncoated minitablets were placed on the bottom of the reactor plate and pretreated in the reactor 229 at $65^{\circ} \mathrm{C}$ and at the pressure of 2 mbar for 24 hours prior to deposition to remove the moisture 230 from the tablets. The thin nanolayers of $\mathrm{TiO}_{2}$ were grown on minitablets from $\mathrm{TiCl}_{4}$ and water at $23165^{\circ} \mathrm{C}$. Nitrogen $\left(\mathrm{N}_{2}\right)$ was used as a carrier and purging gas. The $\mathrm{TiCl}_{4}$ and water were evaporated 232 from the sources at $20^{\circ} \mathrm{C}$. The cycle consisted of a $300 \mathrm{~ms} \mathrm{TiCl} 4$ pulse, $20 \mathrm{~s} \mathrm{~N} 2$ purge, $300 \mathrm{~ms}$ 233 water pulse and $30 \mathrm{~s} \mathrm{~N}_{2}$ purge. The number of ALD cycles was 100, 300 and 500 corresponding 234 to approximately 10, 30 and $50 \mathrm{~nm}$ film thicknesses measured from the silicon reference sample 235 processed in the same runs. Using the approximate coating film thickness $(10,30$ and $50 \mathrm{~nm})$ and 236 the density of $\mathrm{TiO}_{2}\left(4.23 \mathrm{~g} / \mathrm{cm}^{3}\right)$, the amount of $\mathrm{TiO}_{2}$ coating was $0.0042 \mathrm{mg} / \mathrm{cm}^{2}, 0.0127$ $237 \mathrm{mg} / \mathrm{cm}^{2}$ and $0.0212 \mathrm{mg} / \mathrm{cm}^{2}$ corresponding to respective ALD cycles of 100, 300 and 500. After 
238 the coating, the minitablets were collected from the coating chamber and were stored in carefully

239 closed glass vials at RT prior to further analyses.

240

241

242

243

244

245

246

247

248

249

250

251

252

253

254

255

256

257

258

\subsection{Characterization of minitablets}

The surface morphology of uncoated and coated minitablets containing denatonium benzoate was studied using a scanning electron microscope (SEM) (FEI Quanta FEG250, FEI Inc., USA) at the Electron Microscope Unit, Institute of Biotechnology, Helsinki, Finland. Dry platinumcoated samples were scanned using a voltage of $10 \mathrm{kV}$. The surface morphology and surface content analysis for uncoated and $\mathrm{TiO}_{2}$ coated minitablets (500 cycles) were further determined by field emission scanning electron microscope with energy dispersive spectroscopy (SEM-EDS) (Hitachi S-4800 equipped with Oxford INCA 350) at the Department of Chemistry, Helsinki, Finland. The measurements were performed using the voltage of $20 \mathrm{kV}$. Minitablet samples were coated with carbon prior to the measurements.

The height of minitablets was measured with a Sony digital micrometer (Sony Digital Indicator U30-F, Sony, Japan) $(n=10)$. The uniformity of mass and uniformity of content tests were performed according to Ph. Eur. (the tests described in the chapters 2.9.5 and 2.9.6, respectively).

The dissolution of denatonium benzoate minitablets was determined using a modified Ph.Eur. (2.9.3) basket method and apparatus A (Erweka DT6, Erweka GmbH, Germany) (n=4). The dissolution medium was $40 \mathrm{ml}$ of SPB $(\mathrm{pH} 7.6)$ at $37-38^{\circ} \mathrm{C}$. The basket rotation speed was 50

rpm. The samples were collected manually at time periods of 0 and $15 \mathrm{~s}$, and then, after every 30 seconds until the end-point of 6 minutes $15 \mathrm{~s}$. The dissolution sample size for denatonium benzoate containing minitablets was $250 \mu 1$. Prior to HPLC analysis, the samples were 
259 centrifuged $(13,200 \mathrm{rpm} / 5 \mathrm{~min})$ and pipetted into HPLC sampling vials. HPLC analyses were

260 performed within 24 hours after dissolution testing.

261 The disintegration tests of uncoated and coated minitablets were performed using a Sotax DT3

262 tablet disintegration apparatus (Sotax AG, Switzerland). The method was slightly modified for

263 small minitablets from the standard Ph.Eur. tablet disintegration method (2.9.1): the bottom of

264 each testing cylinder was covered with a stainless steel mesh (with an average mesh size 0.2

$265 \mathrm{~mm}$ ) to prevent a small tablet dropping down to the bottom of a test glass beaker.

266 The tablet breaking force (tablet hardness) was determined using a Schleuniger-2E tablet

267 hardness tester (Dr. K. Schleuniger \& Co., Switzerland) with a slight modification compared to

268 the Ph.Eur. method (2.9.8). Instead of determining the load required to crush a tablet

269 diametrically (when placing tablets diametrically onto their flat side between the jaws), tablets

270 were placed diametrically on their edge (belt) between the jaws. This was due to the occasional

271 non-detectability of the load needed to crush the minitablet placed diametrically onto their flat

272 side.

$273 \quad 2.7$ HPLC assays

274 For the uniformity of content and dissolution tests, the samples were analyzed using an Agilent

2751100 series HPLC system (Agilent Technologies, USA) equipped with an UV-Vis detector. The

276 reverse-phase column Zorbax Eclipse Plus C18 (100x4.6 mm, $3.5 \mu \mathrm{m})$ (Agilent Technologies,

277 USA) was utilized in the analyses.

278 The flow rate of eluent mixture of ammonium acetate $(10 \mathrm{mM}, \mathrm{pH} 4.5): \mathrm{ACN}$ was $1 \mathrm{ml} / \mathrm{min}$.

279 Denatonium benzoate was detected at wavelength $210 \mathrm{~nm}$ at $25^{\circ} \mathrm{C}$. For uniformity of content 280 test, the corresponding ratio of the eluents and the retention time of denatonium benzoate was 
$28155: 45$ and $1.8 \mathrm{~min}$ (for the uncoated and the polymer coated minitablets), and 60:40 and 2.4 min

282 (for the uncoated and the $\mathrm{TiO}_{2}$ coated minitablets). For dissolution testing of uncoated and both

283 polymer and $\mathrm{TiO}_{2}$ coated minitablets, the ratio of the eluents was 60:40, and the retention time of

284 denatonium benzoate was $2.4 \mathrm{~min}$.

\subsection{Statistical method of calculation}

286 The Student's t-test (two-tailed distribution, two-sample unequal variance) was used for the

287 calculation of statistical differences between uncoated and coated tablets, these in the results of 288 tablet dissolution, uniformity of tablet mass and denatonium benzoate content, tablet 289 disintegration rate, breaking force and height. Difference between uncoated and coated tablets 290 was considered statistically significant with $\mathrm{p}<0.05$.

\section{3. RESULTS}

\subsection{Content and dimensions of minitablets}

293 Table 2 shows the weight, active ingredient content, disintegration time, breaking force and 294 height of uncoated and coated tablets. For $\mathrm{TiO}_{2}$ coated minitablets, no statistically significant $295(\mathrm{p}<0.05)$ change in the tablet height between uncoated tablets and different coatings of 100, 300 296 or 500 cycles was revealed, though the average tablet height of uncoated tablets was slightly 297 higher than that of coated minitablets. As expected, similarly to tablet height, no changes in the 298 weight of $\mathrm{TiO}_{2}$ coated minitablets or denatonium benzoate content were revealed.

299 Compared to uncoated tablets, a slight decrease in the denatonium benzoate content was 300 observed in polymer film coated tablets, the difference being statistically significant with 301 polymer amounts of 6 and $8 \mathrm{mg} / \mathrm{cm}^{2}$ (Table 2). In comparison to uncoated tablets, film coated 
302 tablets exhibited also an increase in the tablet height and weight, particularly with polymer

303 amounts of 4, 6 and $8 \mathrm{mg} / \mathrm{cm}^{2}$ (tablet height) and with polymer amounts of 2,6 and $8 \mathrm{mg} / \mathrm{cm}^{2}$

304 (tablet weight). The difference between uncoated and polymer coated tablets seemed gradual and

305 in accordance with increased polymer amount. The increase in tablet height with coating of 306 polymer amounts of 2 and $4 \mathrm{mg} / \mathrm{cm}^{2}$ was lower than with polymer amounts 6 and $8 \mathrm{mg} / \mathrm{cm}^{2}$. The

307 highest increase in tablet height and weight was observed with polymer amount of $8 \mathrm{mg} / \mathrm{cm}^{2}$.

308 Tablet height remained relatively unchanged with polymer amount of $2 \mathrm{mg} / \mathrm{cm}^{2}$ when compared

309 to uncoated tablets. However, the average weight of minitablets with coating of $2 \mathrm{mg} / \mathrm{cm}^{2}$

310 decreased in comparison to uncoated minitablets.

\subsection{Minitablet hardness}

312 The $\mathrm{TiO}_{2}$ coating significantly $(\mathrm{p}<0.05)$ decreased the breaking force (hardness) of minitablets in 313 comparison to uncoated minitablets (Table 2). No difference in the breaking force of minitablets

314 between different $\mathrm{TiO}_{2}$ thicknesses (100, 300 and 500 cycles) was revealed.

315 Surprisingly, the breaking force of polymer film coated tablets was also lower than that of 316 uncoated minitablets. No changes in the breaking force were seen between the minitablets coated 317 with polymer concentration of 2 and $4 \mathrm{mg} / \mathrm{cm}^{2}$, and between the minitablets coated with polymer 318 concentration of 6 and $8 \mathrm{mg} / \mathrm{cm}^{2}$.

\subsection{Disintegration in vitro}

320 The disintegration times of uncoated, $\mathrm{TiO}_{2}$ coated, and polymer film coated minitablets are

321 presented in Table 2. Surprisingly, the disintegration time of $\mathrm{TiO}_{2}$ coated minitablets was clearly 322 shorter $(p<0.05)$ than that obtained with uncoated minitablets. No clear differences in the 323 disintegration times between ALD coated tablets (100, 300 and 500 cycles) were revealed as the 
324 disintegration tendency described by the average disintegration time was only slightly decreasing 325 over coating cycles.

326 Surprisingly, the disintegration time of minitablets coated with polymer concentrations of 2 and

$3274 \mathrm{mg} / \mathrm{cm}^{2}$ was shorter in comparison to that of uncoated minitablets. However, minitablets

328 coated with polymer concentration of $4 \mathrm{mg} / \mathrm{cm}^{2}$ disintegrated more slowly than minitablets

329 coated with $2 \mathrm{mg} / \mathrm{cm}^{2}$ polymer concentration. In addition, the disintegration time of minitablets

330 coated with polymer concentration of $6 \mathrm{mg} / \mathrm{cm}^{2}$ was shorter than that of minitablets coated with

331 higher amount $\left(8 \mathrm{mg} / \mathrm{cm}^{2}\right)$ of polymer, but compared with uncoated tablets, the difference with

332 polymer amount of $6 \mathrm{mg} / \mathrm{cm}^{2}$ was not statistically significant. As expected, the disintegration of 333 minitablets containing polymer concentration of $8 \mathrm{mg} / \mathrm{cm}^{2}$ was slower than that of uncoated or 334 other polymer coated tablets of the study. In addition, the minitablets coated with higher polymer 335 concentration of $8 \mathrm{mg} / \mathrm{cm}^{2}$ exhibited a clear lag-time prior to disintegration which was not 336 observed with the $\mathrm{TiO}_{2}$ coated minitablets.

\section{$337 \quad 3.4$ Dissolution in vitro}

338 The dissolution profiles of uncoated, ALD coated $\left(\mathrm{TiO}_{2}\right)$ and polymer film coated minitablets are

339 shown in Fig. 2. With both uncoated and ALD coated minitablets (100, 300 and 500 cycles), the 340 lag-time for denatonium benzoate release was very short (less than 45 seconds). With polymer 341 film coated minitablets, denatonium benzoate release was detected at $45 \mathrm{~s}\left(2,4\right.$ and $\left.6 \mathrm{mg} / \mathrm{cm}^{2}\right)$ 342 and at $1 \mathrm{~min} 15 \mathrm{~s}\left(8 \mathrm{mg} / \mathrm{cm}^{2}\right)$.

343 At 45 seconds, the highest amount of released denatonium benzoate was detected for minitablets 344 containing $2 \mathrm{mg} / \mathrm{cm}^{2}$ and $4 \mathrm{mg} / \mathrm{cm}^{2}$ of coating polymer, while no difference in the released 345 amount of denatonium benzoate was revealed between uncoated and polymer film coated (6 
$\left.346 \mathrm{mg} / \mathrm{cm}^{2}\right)$ minitablets. The polymer film coating of minitablets $\left(2,4\right.$ and $\left.6 \mathrm{mg} / \mathrm{cm}^{2}\right)$ increased the 347 release rate of denatonium benzoate compared to that obtained with uncoated minitablets. 348 However, when the highest amount of coating polymer $\left(8 \mathrm{mg} / \mathrm{cm}^{2}\right)$ was used, the minitablets 349 exhibited a clear delay in the release rate of denatonium benzoate.

$350 \mathrm{TiO}_{2}$ ALD coating on minitablets had only a small effect on the release rate and profile of 351 denatonium benzoate. The slowest release of denatonium benzoate was revealed for 500 cycles

$352 \mathrm{ALD} \mathrm{TiO}_{2}$ coated minitablets. Application of 100 and 300 cycles in the $\mathrm{TiO}_{2} \mathrm{ALD}$ coating of 353 minitablets exhibited only a slight delay in the release rate of denatonium benzoate compared to 354 that obtained with uncoated minitablets.

\subsection{SEM and EDS}

356 The SEM images defining the internal structure and surface morphology of uncoated and coated 357 minitablets are shown in Fig. 3. The SEM-EDS linescans of $\mathrm{TiO}_{2} \mathrm{ALD}$ coated minitablets (500 cycles) are presented in Fig. 4 (intact minitablet) and Fig. 5 (the cross-section of the minitablet). 359 The surface of uncoated minitablet (Fig. 3A) was relatively smooth with dark and white areas, and application of $\mathrm{TiO}_{2}$ ALD coating had virtually no effect on the surface morphology of 361 minitablets (100 cycles in Fig. 3B, 300 cycles in Fig. 3C, and 500 cycles in Fig. 3D). The surface 362 of polymer film coated tablet $\left(2 \mathrm{mg} / \mathrm{cm}^{2}\right.$ in Fig. 3E) revealed rough eroded holes on an uneven 363 tablet surface. Smoother and more even minitablet surfaces were obtained with higher coating 364 polymer concentrations $\left(4 \mathrm{mg} / \mathrm{cm}^{2}\right.$ in Fig. $3 \mathrm{~F}, 6 \mathrm{mg} / \mathrm{cm}^{2}$ in Fig. $3 \mathrm{G}$, and $8 \mathrm{mg} / \mathrm{cm}^{2}$ in Fig. $\left.3 \mathrm{H}\right)$.

365 The SEM-EDS linescan of the $\mathrm{TiO}_{2}$ ALD coated tablet surface revealed the presence of titanium 366 and chlorine on the surface of minitablets (Fig. 4). Moreover, the intensity of the titanium signal 367 seemed to be in accordance with the intensities of elements of calcium and phosphorous (arrows 
368 in Fig. 4 for titanium). With the cut ALD coated minitablet, the intensity of the titanium signal 369 was verified to be exceptionally high on the minitablet surface but decreased rapidly when 370 moving towards the minitablet core (Fig. 5). The penetration of Ti into the structure of the tablet

371 was reaching an approximate depth of $0.2 \mathrm{~mm}$. No titanium was present at the surface of 372 uncoated minitablet (data not shown).

\section{DISCUSSION}

\subsection{Effect of coating method on the properties of minitablets}

375 It is well known that the tablet cores in a conventional fluidized-bed polymer coating process are 376 exposed to a long-term attrition (friction) and collisions resulting in the erosion (even breakage) 377 of tablets during processing. However, the ALD thin coating of tablets with $\mathrm{TiO}_{2}$ in flow type 378 ALD reactor is a much more gentle procedure as the tablets are stationary during coating and 379 thus, are not susceptible to kinetical attrition or collisions similar in fluidized-bed. Thus, no statistically significant differences between uncoated and ALD-coated tablets were revealed for tablet integrity, weight, dimensions, or active drug content.

Surprisingly, however, the breaking force (hardness) and disintegration time of minitablets decreased after ALD coating. This was confusing, especially as in the literature, the ALD coated

384 3-layer tablets have been reported to comply with the specifications for hardness and 385 disintegration time of such dosage forms (Lehtonen et al., 2013). Thus, by contrast to a decrease 386 in the minitablet strength and disintegration, a delay was our more likely expectation, 387 particularly as in theory, the chosen minitablet formulation was thought to support the formation 388 of even, pinhole-free and moisture protective $\mathrm{TiO}_{2} \mathrm{ALD}$ nanolayers. Moreover, in the present 389 study, the experimental composition of minitablet core was considered ideal for ALD thin 
coating, since the presence of the free hydroxyl groups on the surface of MCC in addition to the tablet surface moisture were expected to provide excellent binding sites for $\mathrm{TiCl}_{4}$. This should foster also the formation of homogeneous $\mathrm{TiO}_{2}$ nanolayers on tablet surfaces, voids and crevices, thus reducing the core ability to interact with its surroundings. Moreover, the low deposition temperature of $65^{\circ} \mathrm{C}$ used in the present study was not harmful for the tablet core. In the literature, $\mathrm{TiO}_{2} \mathrm{ALD}$ nanolayering of tablets using $\mathrm{TiCl}_{4}$-water chemistry has been successfully conducted at temperatures even lower than $65^{\circ} \mathrm{C}$ (Lehtonen et al., 2013). The decrease in hardness and disintegration time of minitablets after ALD thin coating can be explained by the fact that pharmaceutical tablets are heterogeneous and porous solid systems, unlike to the substrates traditionally used in ALD thin coating. Moreover, the real ALD film growth mechanism may not be as simple as the theory outlines.

A short interaction between moisture and heterogeneous tablet core components (such as the disintegrant crospovidone or MCC) can cause the microerosion of tablets during an ALD thin coating processing. It is evident that this also induces the creation of new pores, new open intact tablet surfaces, and even new complicated pore networks to the tablet structure. Moreover, the risk for microerosion can increase (especially at low temperatures) because of the liberation of the reaction side product of hydrochloric acid gas, due to $\mathrm{TiCl}_{4}$-water chemistry, adsorbing onto the $\mathrm{TiO}_{2}$ surface (Ritala et al., 1993). As microerosion enlarges, the active surface area of tablets requiring coating also becomes larger. This means that during deposition the exposed new surfaces and pore networks are not likely to be adequately covered by $\mathrm{TiO}_{2}$ nanolayers, simply due to insufficient amount of coating precursor. Thus, microerosion could reduce the layer moisture protecting capability and accelerate the tablet disintegration rate, as well as decrease the tablet hardness. Moreover, in theory, the $\mathrm{TiO}_{2}$ is covalently bonded to the hydroxyl-covered 
413 substrate surface. This could indicate the formation of a completely new chemical component 414 with characteristics totally different from those of the original substance. Also, the variation in 415 the configuration and the level of the hydroxyl-grouped surfaces may complicate the film growth 416 (Ritala et al., 1993). It is evident that areas with uneven and variable level of hydroxyl groups

417 lead to uneven $\mathrm{TiO}_{2}$ layers. Furthermore, the amphoteric chemical behavior of $\mathrm{TiO}_{2}$ surface due 418 to differently orientated and bonded hydroxyl groups on the $\mathrm{TiO}_{2}$ surface (Parfitt, 1976) can lead 419 to additional reactions other than with $\mathrm{TiCl}_{4}$. Low deposition temperatures used in the $\mathrm{TiO}_{2} \mathrm{ALD}$ 420 thin coating can also induce the formation of chemical impurities (residual ions), such as 421 chlorine in ALD thin films originating from $\mathrm{TiCl}_{4}$ precursor ligands (Ritala et al., 1993; Jõgi et 422 al., 2006), and may therefore change the film properties. In addition, at this growth temperature $423 \mathrm{ALD} \mathrm{TiO}_{2}$ thin films are in fact amorphous and may thus have increased intensity for moisture 424 related interactions.

425 The success of $\mathrm{TiO}_{2}$ ALD coating is therefore very much dependent on the tablet core 426 composition, structure and properties. With the present minitablets, phenomena occurring on the 427 heterogeneous tablet surface are hence strongly emphasized. Lehtonen and co-workers (2013) 428 investigated the ALD thin coatings applied to the tablets composed of three-layered 429 pharmaceutical solids containing layers of probiotics and vitamin C. Pharmaceutical tablets are 430 commonly compressed from heterogeneous mixtures. Due to such complex compositions, the 431 coatings applied to the outer surface of tablet cores are exposed to multiple different 432 components, which all have characteristic porosity and interaction capabilities, e.g. with 433 moisture or chemicals. Consequently, the formation of even and pinhole-free $\mathrm{TiO}_{2} \mathrm{ALD}$ 434 nanolayers is greatly affected by the chemical composition of the tablet surface (especially, the 435 excipients rich on hydroxyl groups play a key role), and most likely also by surface moisture. In 
436 other words, the formation and growth of $\mathrm{TiO}_{2}$ thin films is fostered by the surfaces exhibiting

437 very high hydroxyl group density. In addition, the pore density, size and tortuosity of excipients

438 can play a crucial role affecting $\mathrm{TiO}_{2}$ ALD thin films. Depending on the physicochemical

439 characteristics, the active outer surface area of tablet excipients can be very different due to

440 interaction with moisture. Consequently, deep voids or complex pore structures can result in

441 inadequate $\mathrm{TiO}_{2}$ ALD nanolayer formation and growth. Moreover, the formation of intact $\mathrm{TiO}_{2}$

442 ALD coating in tablet pores is most likely to be fostered by the presence of high hydroxyl group

443 density. With the present minitablets, it is evident that excipients and/or denatonium benzoate

444 interact with titanium to different extent and mechanism, thus affecting the formation of

445 nanolayers. Therefore, the reason for the unexpected decrease in the hardness and disintegration

446 time of coated minitablets is most likely the heterogeneity and physicochemical diversity of

447 chemical components in the tablet surface.

448 ALD thin coating has a number of advantages compared to a traditional fluidized-bed film 449 coating method. The $\mathrm{TiO}_{2}$ ALD nanolayering is a gentle procedure in a flow type reactor without 450 any actual collision related impact on tablets. On the contrary, the traditional fluidized-bed 451 coating requires adequate mechanical strength of tablet cores, and appropriate and well-designed 452 tablet compositions. In a fluidized-bed coating process, tablet breakage takes place readily due to 453 a long-term collision and abrasion during fluidization, and due to the elevated temperature and 454 humidity conditions during polymer dispersion spraying and drying. Moreover, particles 455 detached from tablet cores can disturb the formation of even polymer coating and lead to a great 456 variation in an active ingredient content, disintegration and dissolution of tablets. It is also well457 known that traditional polymer film coating can lead to a significant increase in tablet weights 
458 and delay in disintegration times, especially if thick polymer coatings involving high amounts of 459 polymer are used.

460 In our study, fluidized-bed polymer film-coated tablets exhibited a clear decrease in hardness 461 (breaking force), tablet weight and denatonium benzoate content compared to the corresponding 462 uncoated tablets, all mostly related to tablet erosion and breakage during film coating process. 463 The possibility for tablet breakage was expected to be avoided with the inclusion of MCC as a 464 main component in the tablet core formulation. MCC deforms plastically under compression and 465 maximizes the area of inter-particle hydrogen bonding, thus increasing tablet mechanical 466 strength and hardness (Bolhuis and Lerk, 1973). Consequently, no friability test was performed 467 for the MCC containing minitablet cores, and also because the tablet friction during the strong 468 fluidization in the actual fluidized-bed apparatus was considered to provide a more accurate 469 result for tablet friability. MCC can also entrap and hold significant amounts of moisture inside 470 its structure (Zografi and Kontny, 1986), thus making it an excipient of choice for the moisture471 sensitive ingredients in tablets. Its moisture related interactions during processing and drying are 472 also well-documented (Luukkonen, 2001; Luukkonen et al., 2001; Kleinebudde, 1994).

473 In fluidized-bed coating, several process-related conditions (e.g. high humidity, agitation and 474 elevated temperatures during spraying and drying) can affect the final polymer film coated 475 tablets. Using aqueous polymer coating dispersions, the process-induced changes (interactions) 476 are naturally more evident if the tablet cores are composed of amorphous and/or moisture 477 sensitive excipients, e.g. binders (HPC) or disintegrants (crospovidone) (Joshi and Petereit, 478 2013). These changes are also greatly dependent on the duration (time) of a fluidized-bed 479 process. In our study, the most significant process-induced changes were resulted by the decrease 480 of the hardness of minitablets. 
481 We found that the minitablet cores used in the fluidized-bed polymer coating experiments did not

482 completely withstand the process conditions (agitation, high humidity and elevated temperature).

483 This was obviously partly due to the interaction of MCC with moisture, thus leading to a notable

484 decrease in the mechanical strength (hardness) of tablets. This finding is also in good agreement

485 with that reported in the literature (Westermarck et al., 1999). Moreover, the moisture-induced

486 softening and erosion of tablet cores are most likely fostered with the presence of moisture-

487 sensitive HPC and crospovidone in the tablet formulation. The deteriorating influence of

488 moisture and agitation continues until the uniform and intact film coating is formed around

489 tablets. Deterioration of polymer film-coated tablets can continue even after a coating process, if

490 moisture is entrapped inside the tablet core and polymer film.

491 To avoid tablet deterioration related problems with moisture-sensitive tablet formulations,

492 spraying of polymer dispersion in fluidized-bed coating could be started with a small amount of

493 dispersion followed by an immediate drying phase. This kind of spraying protocol is expected to

494 enhance the initial film formation, thus protecting tablet cores from future moisture interactions.

495 In the fluidized-bed film coating of minitablets, it is also important to control and optimize the

496 spraying properties of dispersion such as droplet size in order to avoid over-wetting and

497 subsequent softening of tablet cores. Compared to large-sized tablets, adequate coating of

498 minitablets containing swellable excipients in particular requires higher amounts of coating

499 polymer due to the larger outer surface area of such minitablets (Mehta, 1997). This is even more

500 important with taste masking film coatings where the applicability and efficacy of coating is

501 dependent of film thickness and intactness. One option to overcome the challenge associated

502 with the larger outer surface area of minitablets is to prolong contact time of tablet cores with the 
503 coating polymer dispersion, e.g. by modifying a Wurster column set-up or by increasing a

504 coating process time. However, such changes will impact also the other coating parameters.

\subsection{Taste masking efficacy of minitablets}

506 Sensing the taste requires the initial interaction of the tablet with moisture (saliva), and

507 subsequent tablet disintegration and dissolution of tastant in the oral cavity. The sensory

508 information from the tongue is then instantly transmitted via neural pathways to the brain to be

509 interpreted as taste perception (Thombre, 2004). The requirement of saliva solubility makes the

510 taste a chemical sense. Normally, the physiological $\mathrm{pH}$ of human saliva is neutral varying from

$511 \mathrm{pH} 6$ to $\mathrm{pH} 7.5$ (Tenovuo, 1995). The diet can greatly affect the $\mathrm{pH}$ of saliva. Therefore, the

512 typical $\mathrm{pH}$ of saliva in herbivores and omnivores is more alkaline than the saliva $\mathrm{pH}$ of

513 carnivores.

514 For the evaluation of minitablet dissolution and bitter taste masking efficacy in vitro, we used an

515 aqueous buffer solution $\mathrm{pH} 7.6$ representing the average $\mathrm{pH}$ for the saliva. The expected

516 residence time of tablets in the mouth was also taken into account in a study protocol. In our

517 study, a mouth residence time longer than 45 seconds without detected bitter tasting denatonium

518 benzoate was required for effective minitablet taste masking, as on average, the time period of a

519 solid dosage form to remain in the mouth is relatively short ranging from a few seconds to 30-60

520 seconds (Joshi and Petereit, 2013). In this study, the modified disintegration and dissolution tests

521 of minitablets in vitro were used to indirectly show the taste masking efficacy of the coated

522 tablets. With better mimicking of the soft tissue function and conditions in the mouth and

523 gastrointestinal system, the more gently proceeding tablet dissolution test with results on 
524 detected denatonium benzoate release were emphasized when used as a tool for the rapid taste

525 masking efficacy evaluation purposes of the coated minitablets of the study.

526 The in vitro dissolution results of $\mathrm{TiO}_{2} \mathrm{ALD}$ coated minitablets were in accordance with the in

527 vitro disintegration times of the respective nanolayered minitablets (Fig. 2). The early

528 denatonium benzoate release from both uncoated and $\mathrm{TiO}_{2}$ ALD coated minitablets was

529 observed at $45 \mathrm{~s}$. This suggests that the coating thickness of the present ALD nanolayered

530 minitablets (i.e., the coating cycles of 100, 300 and 500) is not sufficient for the effective taste

531 masking. The present finding is rather surprising since the multiple $\mathrm{TiO}_{2}$ nanolayers were

532 expected to act as effective barriers to prevent water and oxygen penetration, and thus delay the

533 onset of denatonium benzoate release, and moreover, as Lehtonen and co-workers (2013)

534 reported about the delay in the onset of drug release of $\mathrm{TiO}_{2} \mathrm{ALD}$ layered vitamin $\mathrm{C}$ tablets as

535 well as the taste and smell masking of ALD coated fish oil soft gelatin capsules. In our study,

536 only negligible delay in the dissolution of denatonium benzoate from $\mathrm{TiO}_{2}$ coated minitablets

537 was observed, this regarding minitablets coated with 500 coating cycles. Furthermore, no

538 statistical significant difference $(\mathrm{p}>0.05)$ was found between the early-stage dissolution (within

$53945 \mathrm{~s}-1 \mathrm{~min} 15 \mathrm{~s}$ ) of $\mathrm{TiO}_{2}$ ALD layered minitablets compared to uncoated minitablets.

540 We found that the minitablets coated with a polymer amount of $8 \mathrm{mg} / \mathrm{cm}^{2}$ are evidently

541 applicable for the bitter taste masking of denatonium benzoate. The present fluidized-bed coated

542 minitablets exhibited the most extensive delay in the onset of disintegration and dissolution in

543 vitro (Fig. 2 and Table 2). However, it should be emphasized that the dissolution and mechanical

544 properties (softening) of these polymer film coated minitablets are greatly dependent on the

545 fluidized-bed coating conditions. The ALD thin coating method has some advantages over

546 fluidized-bed coating conditions. The ALD nanolayering is a simple and rapid method, and 
547 especially applicable in case of moisture sensitive tablet formulations since tablet cores are not

548 exposed to high humidity conditions similar to those in fluidized-bed coating. Consequently,

549 ALD nanolayering should not affect the physicochemical and pharmaceutical properties of

550 tablets. Our results suggest also that the adequate taste masking efficacy of minitablets could be

551 achieved by increasing the number of coating cycles in the $\mathrm{TiO}_{2}$ ALD nanolayering, as the

552 release of bitter tasting denatonium benzoate was, though only slightly, delayed from minitablets

553 coated with 500 coating cycles. Attention should also be put onto the time required for the

554 precursor pulse, as the unexpected dissolution of $\mathrm{TiO}_{2}$ coated minitablets could be also explained

555 by the pulse of $300 \mathrm{~ms}$ being possibly inadequate for precursors to penetrate into the tablet pores.

556 In addition, the coating materials other than $\mathrm{TiO}_{2}$ or the multilayer ALD coatings could also open

557 new alternatives to single ALD thin coatings for taste masking applications. For example, $\mathrm{Al}_{2} \mathrm{O}_{3}$

558 ALD thin coated tablets have been shown to exhibit faster drug release in water in comparison to

$559 \mathrm{TiO}_{2}$ ALD coated tablets (Lehtonen et al., 2013). The use of an ALD coating system (reactor)

560 other than the flow type one could also provide improvements in taste masking efficiency.

561 Carlsson and co-workers (2016) reported the existence of holes in the ALD coated nanoparticles

562 due to the contact points with other nanoparticles during deposition. In theory, this could be also

563 possible with the present $\mathrm{TiO}_{2}$ ALD thin-coated minitablets, since the exposure of stationary

564 tablet(s) to ALD could lead to disruptions in thin coatings due to the direct contact of the tablet

565 lower surface with an ALD tray. The probability for the formation of uncoated contact points in

566 minitablets, however, is minimal, since the tablets are slightly vibrating in the semifluid state

567 during the flow of ALD coating. Therefore, the major reason for the defects of $\mathrm{TiO}_{2} \mathrm{ALD}$ thin

568 coating (and obviously for a limited taste masking efficacy of minitablets) is the heterogeneous

569 structure of the tablet surface. It is evident that the surface moisture is adequate for ALD and for 
570 the formation of even $\mathrm{TiO}_{2}$ nanolayers, but the affinity of some tablet excipients varies a lot and

571 this can prevent the formation of homogeneous and continuous $\mathrm{TiO}_{2}$ nanolayers.

\section{$572 \quad 4.3$ Surface morphology and content analysis}

573 The surface properties of fluidized-bed film coated minitablets were dependent on the amount of

574 coating polymer used. The minitablets coated with the lowest polymer amount of $2 \mathrm{mg} / \mathrm{cm}^{2}$

575 exhibited a large variation in film coating appearance (quality) due to erosion and tablet breakage

576 (Fig. 3E). The tablets coated with the highest polymer amount of $8 \mathrm{mg} / \mathrm{cm}^{2}$ had an intact and

577 smooth surface (Fig. 3H). The actual coating thickness of polymer coated tablets ranged from

578 some tenths of micrometers $\left(2 \mathrm{mg} / \mathrm{cm}^{2}\right)$ to a few hundred micrometers $\left(8 \mathrm{mg} / \mathrm{cm}^{2}\right)$. Such great

579 variation in coating thicknesses (and intactness) explains also the changes in physical and 580 pharmaceutical properties of minitablets coated with different amounts of polymer.

581 Since SEM did not permit the nanometer-scale analysis of the ALD nanolayered minitablets, the

582 surface of an uncoated and a $\mathrm{TiO} 2$ coated (500 cycles) minitablet was analyzed also with SEM-

583 EDS. This analysis (elemental mapping) was primarily carried out to explain the accelerated

584 disintegration and dissolution behavior of ALD thin-coated minitablets. The presence of Ti

585 signal on the porous ALD coated minitablet surface can be considered as an important 586 verification on the successful nanolayer formation onto the surface of minitablets. In accordance

587 to the theory on hermetic ALD layers on porous minitablets, the SEM-EDS results suggested that 588 the $\mathrm{TiO}_{2}$ layer was integral and indeed entirely covering the various areas over the linescan on 589 the minitablet surface leaving no disrupted areas in the coating. Moreover, in agreement with our 590 theory of ALD thin coating on porous tablets, the $\mathrm{TiO}_{2}$ precursors seem to diffuse into the voids 591 and pores of minitablets with the estimated diffusion length of approximately 100-200 $\mu \mathrm{m}$. This 
592 finding supports the view that the ALD thin coating of minitablets can be successfully 593 performed. The major limitation of the presented coated substrates, however, was that they did 594 not permit us to analyze the interface between the minitablet core and nanolayer, and thus to 595 determine the actual thickness of titanium coating. Lehtonen and co-workers (2013) used SEM to 596 measure a nanometer scale $\mathrm{Al}_{2} \mathrm{O}_{3}(10 \mathrm{~nm})+\mathrm{TiO}_{2}(10 \mathrm{~nm})$ layer thickness of fish oil containing 597 soft gel gelatin capsules. In our study, however, the analysis of the interface of tablet core and 598 nanolayers, and coating thickness are much more challenging due to the porous structure of 599 tablet surface compared to that of soft gelatin capsule. Nevertheless, this issue could be 600 overcome by the verification of integral titanium layer on minitablets combined with the known 601 theoretical nanocoating thickness.

602 In addition to titanium, traces of chlorine were also present on $\mathrm{TiO}_{2} \mathrm{ALD}$ coated minitablet 603 surface. This is obviously due to a porous substrate and low ALD coating temperature of $65^{\circ} \mathrm{C}$ 604 which is known to leave residuals of incompletely reacted $\mathrm{TiCl}_{4}$ on surfaces (Ritala et al., 1993). 605 The residuals present could be minimized by increasing the ALD coating temperature up to and 606 even over $200^{\circ} \mathrm{C}$ (Ritala et al., 1993). Many active pharmaceutical substances and excipients, 607 however, are thermally sensitive, which limits the use of very high handling temperatures during 608 ALD thin coating. Further studies will be needed on the formation and role of precursor residuals 609 (including safety and toxicity) in ALD nanolayers.

610 The SEM-EDS morphological and elemental analysis revealed that the surface of uncoated 611 minitablets is porous and heterogeneous due to the multiple excipients used for formulating the 612 tablet cores. The surface of uncoated minitablets (cores) was covered with the grey-white and 613 dark areas (spots), which were still clearly visible in TiO2 ALD coated tablets. The bright areas 614 revealed a more intense signal for phosphorous and calcium over dark areas. The presence of 
615 phosphorous and calcium refers to the calcium hydrogen phosphate dihydrate used as an 616 excipient in the minitablet composition. Surprisingly, the behavior of phosphorous and calcium

617 seemed to be followed by an increase in the manifestation for titanium. The difference in

618 titanium intensity between white and dark areas was found to be notable and in contrast to other

619 surface elements. The intensity of titanium was clearly the highest in the white surface areas

620 together with phosphorous and calcium.

621 The present finding is considered as significant. The high intensity of titanium signals indicates

622 that certain pharmaceutical excipients (such as in our study calcium hydrogen phosphate 623 dihydrate) can be coated (nanolayered) with $\mathrm{TiO}_{2}$ more easily than others. The differences in the 624 intensity of titanium signals can also indicate the uneven formation of nanolayers on the 625 heterogeneous surface of minitablets, thus resulting in the limited moisture protection and 626 changes of the performance of ALD coated minitablets. However, the formation of uneven 627 nanolayers, can be argued since titanium was also detected in the surface pores of the minitablet 628 cores, and may therefore not be seen there at the equal levels found in the calcium and 629 phosphorous rich surfaces. Further studies will be needed on using $\mathrm{TiO}_{2}$ nanolayers with both 630 active pharmaceutical substances and excipients.

\section{5. CONCLUSIONS}

632 ALD provides a simple and rapid method for the ultrathin coating (nanolayering) of minitablets. 633 It is evident that the level of agitation and collision forces between tablets in ALD are minor 634 compared to polymer coating in a fluidized-bed, not to mention that the method is relatively 635 simple compared to the conventional and challenging polymer film coating process. The ALD 636 thin coating method has both advantages and limitations over traditional film coating methods. 
637 The ALD thin coating is a rapid process since no spraying and drying steps are needed.

638 However, the unexpected changes in the $\mathrm{TiO}_{2} \mathrm{ALD}$ coated minitablet mechanical properties seen

639 as an accelerated tablet disintegration rate and decreased tablet hardness may challenge its

640 feasibility in the coating of heterogeneous substrates composed of substances with different

641 chemical characteristics and reaction ability, until a thorough understanding on possible

642 phenomena taking place on tablet surface and within used diverse and physicochemically

643 variable excipients during ALD process is established. Therefore, the major challenges of ALD

644 thin coating are related to the sufficient taste masking capacity and interactions of

645 coating/layering material $\left(\mathrm{TiO}_{2}\right)$ with the excipients used in the tablet core. More research is

646 needed to clarify the potential of ALD in tablet coating applications. For example, coating

647 materials other than $\mathrm{TiO}_{2}$, the effects of the number of coating cycles and the length of the

648 precursor pulses on the taste masking are suggested for further study.

649 ACKNOWLEDGEMENTS

650 The authors would like to thank Research Technician Mervi Lindman from the Electron

651 Microscopy Unit of University of Helsinki for all her expertise in SEM imaging of minitablets.

\section{FUNDING}

653 This research did not receive any specific grant from funding agencies in the public, commercial, 654 or not-for-profit sectors. 


\section{REFERENCES}

656 Ayenew, Z., Puri, V., Kumar, L., Bansal, A.K., 2009. Trends in pharmaceutical taste masking 657 technologies: A patent review. Recent Pat. Drug Deliv. Formul. 3, 26-39.

658 Bolhuis, G.K., Lerk, C.H., 1973. Comparative evaluation of excipients foe direct compression, I.

659 Pharm. Weekbl. 108, 469-481.

660 Bruce, H.F., Sheskey, P.J., Garcia-Todd, P., Felton, L.A., 2011. Novel low-molecular-weight 661 hypromellose polymeric films for aqueous film coating applications. Drug Dev. Ind. Pharm. 37, $662 \quad 1439-1445$.

663 Carlsson, J-O., Johansson, A., Rooth, M., 2016. A solid nanoparticle with inorganic coating. U.S. 664 Patent 0081945 A1.

665 Evonik Industries. Eudragit ${ }^{\circledR}$ Application Guidelines, $11^{\text {th }}$ Ed. 2009.

666 Felton, L.A., 2007. Characterization of coating systems. AAPS PharmSciTech 8, 112.

667 George, S., 2010. Atomic layer deposition: an overview. Chem. Rev. 110, 111-131.

668 Hoppu, P., Kaariainen, T., Kaariainen, M-L., Turunen, A., 2015. Method for coating 669 pharmaceutical substrates. U.S. Patent 0250731 A1.

670 Joshi, S., Petereit, H-U., 2013. Film coatings for taste masking and moisture protection. Int. J. 671 Pharm. 457, 395-406.

672 Jõgi, I., Aarik, J., Laan, M., Lu, J., Kukli, K., Käämbre, H., Sajavaara, T., Uustare, T., 2006. 673 Effect of preparation conditions on properties of atomic layer deposited $\mathrm{TiO}_{2}$ films in $\mathrm{Mo}^{-} \mathrm{TiO}_{2}-$ 674 Al stacks. Thin Solid Films 510, 39-47. 
675 Kleinebudde, P., 1994. Shrinking and swelling properties of pellets containing microcrystalline 676 cellulose and low substituted hydroxypropylcellulose: I. Shrinking properties. Int. J. Pharm. 109, $677 \quad 209-219$.

678 Lehmann. K., 1997. Chemistry and application properties of polymethacrylate coating systems, 679 in: McGinity, J., (Ed.), Aqueous Polymeric Coatings for Pharmaceutical Dosage Forms, $2^{\text {nd }}$ Ed. 680 Marcel-Dekker, Inc., New York, USA, pp. 101-176.

681 Lehtonen, L., Bley, H., Van Der Heyden, L., 2013. Coated solid pharmaceutical preparation. 682 U.S. Patent 0337056 A1.

683 Luukkonen, P., 2001. Rheological properties and the state of water of microcrystalline cellulose 684 and silicified microcrystalline cellulose wet masses. $\mathrm{PhD}$ Thesis, University of Helsinki, Finland.

685 Luukkonen, P., Maloney, T., Rantanen, J., Paulapuro, H., Yliruusi, J., 2001. Microcrystalline 686 cellulose-water interaction - a novel approach using thermoporosimetry. Pharm. Res. 18, 15626871569.

688 Mehta, A., 1997. Processing and equipment considerations for aqueous coatings, in: McGinity, 689 J., (Ed.), Aqueous Polymeric Coatings for Pharmaceutical Dosage Forms, 2 ${ }^{\text {nd }}$ Ed. Marcel690 Dekker, Inc., New York, USA, pp. 287-326.

691 Parfitt, G.D., 1976. The surface of titanium dioxide. Progr. Surf. Membrane Sci. 11, 181-226.

692 Pearnchop, N., Siepmann, J., Bodmeier, R., 2003. Pharmaceutical applications of shellac: 693 moisture-protective and taste-masking coatings and extended-release matrix tablets. Drug Dev. 694 Ind. Pharm. 29, 925-938. 
695 Puurunen, R., 2005. Surface chemistry of atomic layer deposition: A case study for the 696 trimethylaluminum/water process. J. Appl. Phys. 97, 121301-121352.

697 Ritala, M., Leskelä, M., 2002. Atomic layer deposition, in: Nalwa, H.S. (Ed.), Handbook of Thin 698 Film Materials, Volume I: Deposition and Processing of Thin Films. Academic Press, San 699 Diego, USA, pp. 103-159.

700 Ritala, M., Leskelä, M., Nykänen, E., Soininen, P., Niinistö, L., 1993. Growth of titanium 701 dioxide thin films by atomic layer epitaxy. Thin Solid Films 225, 288-295.

702 Siepmann, J., Bodmeier, R., McGinity, J., 2013. Progress in film coating. Int. J. Pharm. 457, 361.

703 Sohi, H., Sultana, Y., Khar, R.K., 2004. Taste masking technologies in oral pharmaceuticals:

704 Recent developments and approaches. Drug Dev. Ind. Pharm. 30, 429-448.

705 Tenovuo, J., 1995. Sylki diagnostisena nesteenä. Duodecim. 111, 1355-1359.

706 Thombre, A.G., 2004. Oral delivery of medications to companion animals: palatability 707 considerations. Adv. Drug Deliv. Rev. 56, 1399-1413.

708 Westermarck, S., Juppo, A.M., Kervinen, L., Yliruusi, J., 1999. Microcrystalline cellulose and its 709 microstructure in pharmaceutical processing. Eur. J. Pharm. Biopharm. 48, 199-206.

710 Zografi, G., Kontny, M.J., 1986. The interactions of water with cellulose- and starch-derived 711 pharmaceutical excipients. Pharm. Res. 3, 187-194. 
Captions to illustrations:

714 Fig. 1. The theoretical formation of $\mathrm{TiO}_{2}$ nanolayers via the chemical reaction between water and $715 \mathrm{TiCl}_{4}$ in atomic layer deposition (ALD) thin-coating process. In ALD involving metal oxides, a 716 cycle of surface saturation takes place through two reaction steps performed commonly in 717 vacuum at controlled temperature. During step one (the first half cycle) the metal precursor such 718 as $\mathrm{TiCl}_{4}$ vapor, pulsed to the coating chamber, is allowed to react with the free hydroxyl groups 719 on the substrate surface. The chemical pulse therefore saturates the surface with Ti containing groups through molecular bonds. During step two (the second half cycle) the molecularly bonded 721 structures on the substrate surface react with the oxygen precursor (water vapor) and form the 722 first nanolayer of $\mathrm{TiO}_{2}$. Between the steps, a purge of inert gas $\left(\mathrm{N}_{2}\right)$ is applied to the chamber to 723 remove the possible excess of precursor and reaction by-products. The film thickness is 724 controlled by repeating the number of reaction cycles to reach the desired coating.

725 Fig. 2. Dissolution profiles of uncoated minitablets, $\mathrm{TiO}_{2}$ ALD (100, 300 and 500 cycles) thin726 coated and fluidized-bed polymer film coated $\left(2,4,6\right.$ and $8 \mathrm{mg} / \mathrm{cm}^{2}$ of polymer) minitablets $727(n=4)$. The dissolution curves describe the cumulative release of denatonium benzoate from 728 minitablets.

729 Fig. 3. Scanning electron microscopy (SEM) images on uncoated minitablet (Fig. 3A), $\mathrm{TIO}_{2}$ 730 ALD thin-coated minitablets (100, 300 and 500 cycles; Fig. 3B-D, respectively) and fluidized731 bed polymer film coated minitablets $\left(2,4,6\right.$ and $8 \mathrm{mg} / \mathrm{cm}^{2}$; Fig. 3E-H, respectively).

732 Fig. 4. Scanning electron microscopy-energy dispersive spectroscopy (SEM-EDS) image on the 733 intact $\mathrm{TiO}_{2}$ ALD thin-coated minitablet (500 cycles). Arrows indicate the presence of titanium on 734 the ALD thin-coated minitablet surface followed by signals on calcium and phosphorous.

735 Fig. 5. SEM-EDS image on the cross-section of $\mathrm{TiO}_{2}$ ALD thin-coated minitablet (500 cycles). 
736 Table 1. Used parameters for minitablet polymer coating.

\begin{tabular}{|l|l|}
\hline Parameter & Value \\
\hline Nozzle diameter & $0.5 \mathrm{~mm}$ \\
\hline Atomizing air pressure & $1.1 \mathrm{bar}$ \\
\hline Inlet air volume & $12.5 \mathrm{l} / \mathrm{s}$ \\
\hline Inlet air temperature & $40-50^{\circ} \mathrm{C}$ \\
\hline Spraying rate & $2.2 \mathrm{~g} / \mathrm{min}$ \\
\hline
\end{tabular}

737 
738 Table 2. Results of the tests performed on both uncoated, and for $\mathrm{TiO}_{2}$ and EPO coated 739 minitablets of the study. Statistically significant $(\mathrm{p}<0.05)$ difference between uncoated and 740 coated tablet is marked in bold.

\begin{tabular}{|c|c|c|c|c|c|}
\hline Tablet & $\begin{array}{c}\text { Uniformity } \\
\text { of mass } \\
\text { Average } \pm \text { SD } \\
\text { (mg) }\end{array}$ & $\begin{array}{c}\text { Uniformity } \\
\text { of content } \\
\text { Average } \pm \text { SD } \\
\text { (mg) }\end{array}$ & $\begin{array}{c}\text { Disintegration } \\
\text { rate } \\
\text { Average } \pm \text { SD } \\
\text { (s) }\end{array}$ & $\begin{array}{c}\text { Tablet } \\
\text { breaking force } \\
\text { Average } \pm \text { SD } \\
\text { (N) }\end{array}$ & $\begin{array}{c}\text { Tablet } \\
\text { height } \\
\text { Average } \pm \text { SD } \\
(\mathbf{m m})\end{array}$ \\
\hline $\begin{array}{c}\text { Batch I } \\
\text { Uncoated }\end{array}$ & $23.0 \pm 0.4$ & $8.6 \pm 0.4$ & $48 \pm 3$ & $50 \pm 4$ & $2.42 \pm 0.02$ \\
\hline $\begin{array}{c}\mathrm{TiO}_{2} \text { coated, } \\
100 \text { cycles }\end{array}$ & $22.7 \pm 0.3$ & $8.3 \pm 0.4$ & $23 \pm 2$ & $45 \pm 2$ & $2.41 \pm 0.03$ \\
\hline $\begin{array}{c}\mathrm{TiO}_{2} \text { coated, } \\
300 \text { cycles }\end{array}$ & $22.8 \pm 0.4$ & $8.5 \pm 0.2$ & $20 \pm 6$ & $43 \pm 3$ & $2.40 \pm 0.02$ \\
\hline $\begin{array}{c}\mathrm{TiO}_{2} \text { coated, } \\
500 \text { cycles }\end{array}$ & $22.7 \pm 0.4$ & $8.7 \pm 0.3$ & $19 \pm 5$ & $44 \pm 4$ & $2.39 \pm 0.02$ \\
\hline $\begin{array}{c}\text { Batch II } \\
\text { Uncoated }\end{array}$ & $24.5 \pm 0.5$ & $10.0 \pm 0.8$ & $44 \pm 8$ & $61 \pm 7$ & $2.46 \pm 0.05$ \\
\hline $\begin{array}{l}\text { EPO coated, } \\
2 \mathrm{mg} / \mathrm{cm}^{2}\end{array}$ & $23.9 \pm 0.5$ & $9.8 \pm 0.9$ & $16 \pm 2$ & $44 \pm 3$ & $2.46 \pm 0.02$ \\
\hline $\begin{array}{l}\text { EPO coated, } \\
4 \mathrm{mg} / \mathrm{cm}^{2}\end{array}$ & $24.8 \pm 0.6$ & $9.6 \pm 0.6$ & $25 \pm 3$ & $44 \pm 4$ & $2.51 \pm 0.04$ \\
\hline
\end{tabular}




\begin{tabular}{|c|c|c|c|c|c|}
\hline $\begin{array}{l}\text { Batch III } \\
\text { Uncoated }\end{array}$ & $23.3 \pm 0.5$ & $10.0 \pm 0.6$ & $31 \pm 3$ & $51 \pm 3$ & $2.40 \pm 0.03$ \\
\hline $\begin{array}{l}\text { EPO coated, } \\
6 \mathrm{mg} / \mathrm{cm}^{2}\end{array}$ & $24.4 \pm 0.6$ & $9.3 \pm 0.4$ & $29 \pm 4$ & $37 \pm 2$ & $2.49 \pm 0.03$ \\
\hline $\begin{array}{c}\text { EPO coated, } \\
8 \mathrm{mg} / \mathrm{cm}^{2}\end{array}$ & $25.5 \pm 0.6$ & $9.6 \pm 0.8$ & $50 \pm 3$ & $41 \pm 3$ & $2.54 \pm 0.02$ \\
\hline
\end{tabular}

741 